\title{
The SERI-ARVO Meeting and future challenges of ophthalmic research in Asia
}

\section{T Y Wong, D T Tan}

\section{Indication of exciting times}

T he progress of ophthalmology in Asia has paralleled much of its socioeconomic evolution. During the early years of a nation's development, scarce resources of a country were usually concentrated on building the basic healthcare infrastructure and services needed to cater for the general population. Over time, with increasing affluence and improved health care, resources could be channelled elsewhere towards developing other goals. One such development is the emergence of ophthalmic research in Asia. As in Western societies, clinical and basic science research is increasingly seen as being an integral component of a well balanced portfolio in many Asian healthcare institutions. This is reflected by the increasing number of high quality publications in leading eye journals that originate from Asia.

Although there are several clinical meetings in ophthalmology held on a regular basis in Asia, there are few large forums in this region that bring together vision and ophthalmic scientists to share their work. It was with this in mind that the Singapore Eye Research Institute (SERI), in direct collaboration with the Association of Research in Vision and Ophthalmology (ARVO) in the United States, organised the SERI-ARVO Meeting in Singapore in February 2003. The meeting covered both clinical research in ophthalmology and basic research in the visual sciences, with specific emphasis on eye diseases relevant to Asians. Several papers in this issue of the $B J O$ are expedited presentations from the SERIARVO meeting.

In addition to the scientific symposia, pre-SERI-ARVO meeting workshops were held to provide an introduction to research methodology. These included a workshop on "Understanding basic science research," which covered principles of basic science research and an introduction to common techniques used in genomics, proteonomics, molecular biology, stem cell research, and ocular immunology. Another workshop, "How to design and conduct clinical research," introduced the clinical investigation

method, with a focus on the design, conduct, and analysis of clinical trials, surveys, and other epidemiological studies relevant to ophthalmology. The third workshop, "How to write a successful grant and scientific paper," provided a broad overview of practical skills in writing a research grant, getting the paper published, ethical issues, and the evidence based approach. These workshops were well attended by both junior and senior ophthalmologists and vision scientists in the region.

\section{Causes of blindness and the spectrum of eye diseases in Asia appear to be different, which suggests that traditional approaches to treatment may not be appropriate}

The success of the SERI-ARVO Meeting is an indication of exciting times for ophthalmic research in Asia. However, several key issues challenge its continued development. What are these? Firstly, one of the current problems faced by many Asian countries is that most institutions and departments, and their heads, still place the highest priority on clinical service, and emphasise high clinical volume and subspecialty expertise as key prerequisites for staff promotions. Thus, the importance of clinical service and teaching frequently consigns research to a low priority on the hospital and departmental agenda. Some clinical heads do not appreciate the effort that a publication in a top journal entails, and feel that research can be done during the lunch hour or after seeing a full load of patients. The concept that high impact research can be performed as a "part time hobby" must change. Asian institutions and hospitals must realise that a well regarded research programme adds incalculable value to their department's reputation and brand name and may, in fact, drive their clinical service. Patients flock to famous ophthalmic institutions in the United States (for example, Wilmer Ophthalmological Institute) and Europe (for example, Moorfields Eye
Hospital) partly based on their high impact, leading edge research.

A second problem is the lack of a core nucleus of clinician-scientists interested in pursuing high quality ophthalmic research, particularly basic science research, in most Asian institutions. This may be somewhat surprising because in the past few decades, many young ophthalmologists in training in Asia are sent overseas to the United States or Europe for further fellowship attachments. These trainees are often required to conduct research as part of their clinical training, and will have been exposed to the rigorous research methods in their supervisors' laboratories, and may come home having published in prestigious journals. However, although young Asian ophthalmologists clearly have the ability to perform high quality research in the right environment overseas, their productivity diminishes when they return home. The reasons for this infertile research soil in Asia are multifaceted and complicated. One aspect could be a lack of protected time wherein young potential clinicianscientists are overwhelmed by demands of patient care and clinical responsibilities on their return. Budding junior ophthalmologist-researchers are also given the impression that research may be "professional suicide" by their clinician colleagues. The clinician-scientist route is not yet perceived as a viable and sustainable career track. In this aspect, Asian countries should look into the success of young faculty and investigator schemes in Western countries (for example, the $\mathrm{K}$ awards from the $\mathrm{Na}$ tional Institutes of Health in the United States and the Wellcome Trust Career Development Programmes in the United Kingdom). ${ }^{1}$

Finally, in Asia, the ethical conduct of research remains erratic. ${ }^{2-4}$ It is not clear that the informed consent, the foundation of ethical human clinical research, is properly administered appropriately in all countries. ${ }^{5}$ New problems raised by the increasing use of large scale human tissue sample collection for genetic research will pose a serious challenge in Asia, as it has been for many years in the West. Issues relating to human rights, privacy, intellectual property rights, and appropriate relationship between academia and commerce will have broad implications for the conduct of biomedical research. Another source of concern is the issue of animal rights in Asia. ${ }^{6}$ These and other problems must be quickly and decisively addressed by the leaders of the ophthalmic research community in Asia for the future development of research in this region.

Asia offers unique opportunities for ophthalmic research. The two most populous countries in the world, China and India, still have one of the higher 
rates of blindness and visual impairment. ${ }^{7}$ Additionally, the causes of blindness and the spectrum of eye diseases in this region appear to be different, which suggests that traditional approaches to treatment may not be appropriate. For example, myopia ${ }^{89}$ and angle closure glaucoma ${ }^{10} 11$ are more common in east Asians than in similarly aged people in the West. Thus, Asia offers researchers the clinical material to focus on these diseases. Ultimately, this will afford a clearer understanding of the aetiology, pathogenesis and natural history, and novel strategies for treatment and prevention of the major blinding diseases in Asia.

Br J Ophthalmol 2003;87:379-380

\section{Authors' affiliations}

T Y Wong, D T Tan, Singapore National Eye Center and Singapore Eye Research Institute, Singapore and Department of Ophthalmology, National University of Singapore

Correspondence to: Tien Yin Wong, FRCSE, $\mathrm{MPH}, \mathrm{PhD}$, Department of Ophthalmology, National University of Singapore, 10 Kent Ridge Crescent, Singapore; ophwty@nus.edu.sg

\section{REFERENCES}

1 Weinreb RN. Clinician-scientists in ophthalmology. Arch Ophthalmol 2001;119:277-9

2 Angell $M$. The ethics of clinical research in the Third World. N Engl J Med 1997;337:847-9.

3 Varmus H, Satcher D. Ethical complexities of conducting research in developing countries. N Engl J Med 1997;337:1003-5.

4 Ono S, Kodama Y, Nagao T, et al. The quality of conduct in Japanese clinical trials: deficiencies found in GCP inspections. Control Clin Trials 2002;23:29-41.

5 Achrekar A, Gupta R. Informed consent for a clinical trial in Thailand. N Engl J Med 1998;339:1331-2.

6 Kumar S. Animal rights activist is removed from government committee. $B M$ 2003;326:68.

7 Keeffe JE, Konyama K, Taylor HR. Vision impairment in the Pacific region. $\mathrm{Br} J$ Ophthalmol 2002;86:605-10

8 Wong TY, Foster PJ, Hee J, et al. Prevalence and risk factors for refractive errors in adult Chinese in Singapore. Invest Ophthalmol Vis Sci 2000;41:2486-94.

9 Seet B, Wong TY, Tan DTH, et al. Myopia in Singapore: taking a public health approach. Br J Ophthalmol 2001;85:521-6.

10 Foster PJ, Johnson GJ. Glaucoma in China: how big is the problem? Br J Ophthalmol 2001;85: 1277-82.

11 Quigley HA, Congdon NG, Friedman DS. Glaucoma in China (and worldwide): changes in established thinking will decrease preventable blindness. Br J Ophthalmol 2001;85:1271-2.

\section{Primary care in ophthalmology}

\section{J Thompson}

\section{Seeing the broader picture}

$\mathrm{R}^{\mathrm{s}}$ ad and colleagues have presented us, in this issue of $B J O$ (p 493), with an interesting and thorough review of primary care especially as it applies to ophthalmology in the United Kingdom. In their far ranging article the authors consider topics such as the history and definitions of primary care, the attributes of good primary care, and the primary-secondary interface. The review accurately portrays the complexity of primary healthcare provision and implicitly raises the question of how such a complex system can be planned, managed, or evaluated. The review will be particularly helpful when health service researchers need to view ophthalmic primary care as a whole because it is not sufficient for them merely to concentrate on one specific aspect.

Most health service research concentrates on the qualities of some small part of healthcare provision, perhaps suggesting how that particular aspect could be improved. While important, this approach sometimes ignores the knock-on effect that changing one part of a service will have on other provision. The coverage of diabetic retinopathy screening schemes in England and Wales was recently audited with the aim of describing the characteristics of services that are best at reaching people with diabetes. ${ }^{1}$ One striking feature of the audit was the way that general practitioners tried to compensate for the absence of an organised scheme by performing more eye checks themselves. Introducing an organised diabetic retinopathy screen will therefore have wider repercussions. Not only will tests given by specialists tend to be more reliable, but general practitioners will become less involved in giving eye checks, allowing them more time for other aspects of their work; this might be positive, but the withdrawal from eye screening could reduce their contact with their diabetic patients and so impact on the total package of care that they provide. Whenever change is advocated for one part of primary care, the impact on the service as a whole also needs to be considered, because changes tend to ripple through. Although obvious, this is frequently ignored when research findings are reported and implemented. Riad et al's review may help researchers who are looking into a particular aspect of ophthalmic primary care to place their work in the broader context.

Those of us who study particular aspects of primary care provision must guard against missing the impact on the service as a whole
As part of the audit of coverage the process of diabetic retinopathy screening was divided into its component parts; identifying people with diabetes, coverage of the screen, the quality of the test, and treatment. ${ }^{2}$ Each of those components has been the subject of much separate research and by bringing together the information on the various components it might be possible to suggest the "ideal" scheme. Unfortunately, reality is more complex than that and the various components may interact with one another. Perhaps increasing the coverage would put added pressure on the screening services so that the sensitivity or specificity of the test would decline. In such circumstances a simpler screening method that is less sensitive to the pressure of numbers of patients might be preferable to a "high tech" solution, even if research demonstrates the theoretical benefits of the latter. Although a lot can be learned from studying parts of the service in isolation, at some stage the programme as a whole needs to be considered. Eventually, more studies will be conducted that specifically relate to the interactions between parts of primary care so that models of the whole system can be developed, but in the meantime the review of Riad et al will help us think about these issues informally.

The difficulties experienced by those of us who are interested in healthcare evaluation are mirrored in the extreme difficulty that is currently being experienced by those trying to manage the NHS. Most ophthalmologists will be familiar with the way that management targets, genuinely aimed at improving one part of the service, end up causing unanticipated repercussions elsewhere. Thus, moving resources to control waiting times for first appointments affects the service's ability to provide follow up 
care. A centrally planned healthcare system has all the problems of a centrally planned economy. Ironically, the NHS used to be lightly managed with care provided by semiautonomous ethically motivated consultants and general practitioners; with proper financing this might actually be one of the best ways of organising a non-fee based service. Management was introduced to control costs but resulted in the more centralised and bureaucratic system that government is now trying to unravel. Primary care is seen as leading the new NHS and this strategic position makes evaluation even more complex, as the primary care services might need to do things to drive the NHS that do not necessarily improve the quality of primary care itself.

Just as scientists who study disease at a cellular level need to guard against missing the impact on the person, so those of us who study particular aspects of primary care provision must guard against missing the impact on the service as a whole. Riad et al's review may help us all in that.

Br J Ophthalmol 2003;87:380-381

\section{Author's affiliations}

J Thompson, University of Leicester, Leicester, UK; tri@le.ac.uk

\section{REFERENCES}

1 Wilson AD, Baker R, Thompson JR, et al. Coverage in screening for diabetic medicine: Results froma a national survey in England and Wales. Diabet Med (in press).

2 Grimshaw GM, Baker R, Wilson $A D$, et al. Screening for diabetic retinopathy: towards a model for the evaluation and planning of screening programmes. J Clinical Excellence $1999 ; 1: 41-6$.

\section{Behçet's syndrome}

\section{R Stanford}

\section{New treatments for an old disease}

$\mathrm{B}$ ehçet's syndrome, a major cause of posterior uveitis and visual loss in the Third World, has probably existed for more than 4000 years. It is a multisystem, inflammatory disorder whose principal manifestations are oral and genital aphthosis as well as inflammation of the eye, skin, and joints. The principal causes of death are from vascular and neurological involvement. No aetiological agent has yet been identified for the disease; current evidence suggests that the normal flora of mucosal tracts induce immunological hyper-reactivity in genetically predisposed individuals.

The visual prognosis in patients with Behçet's disease is poor, the principal cause of visual loss being consecutive inflammatory ischaemic retinal vein occlusions and macular oedema. Treatment is directed at suppressing the inflammatory response using corticosteroids and a variety of second line immunosuppressive agents. Despite different treatment regimens used in different countries the visual prognosis is much the same, with a hard core of $15-20 \%$ of patients seemingly resistant to therapy and progressing relentlessly to blindness. ${ }^{1}$ Randomised trials have shown some efficacy of both azathioprine and cyclosporin A, but the results are only reported in the short term and there is little reliable evidence that they have a substantial effect long term. In this issue of the $B J O$, Kötter et al ( $\mathrm{p} 423$ ) report their experience using subcutaneous injections of recombinant human interferon alfa-2a for the treatment of this disease in a group of patients resistant to high dose corticosteroids. Remarkably, $40 \%$ of their patients were disease free off all medication at 3 years (range 12-72 months), while others were maintained at a low dose of interferon alfa-2a and prednisolone. Reported side effects included a universal flu-like illness at the start of treatment, with leucopenia $(40 \%)$, hair loss $(24 \%)$, itching ( $10 \%)$, and depression ( $8 \%$ ) also occurring. All side effects were reversible with a reduction in dosage. The development of autoantibodies was noted during treatment, but clinically detectable autoimmune disease (thyroiditis) only occurred in two patients. Certainly, the side effect profile was considerably better than that associated with other immunosuppressive drugs, a fact reflected by the low non-compliance rate in the present study. These results are extremely promising, especially considering that the entry criteria for patients in the study was failure to control disease at doses of prednisolone of $1 \mathrm{mg} / \mathrm{kg} /$ day or relapse when the dose was reduced to less than $30 \mathrm{mg} /$ day. Although there are caveats to the study, in that it was an open, non-randomised and uncontrolled trial, the results fully justify the development of prospective, randomised studies. $^{2}$ Recent pharmaceutical advances have resulted in a formulation of interferon alfa that need only be given once a week (IFN-PEG), and other even longer acting formulations are in the pipeline, which hopefully will improve patient acceptability and the side effect profile even further.

It is currently unclear how interferon alfa works in this clinical scenario. The drug is widely used as a myelosuppressive agent in the treatment of a variety of haematological malignancies and has found a place in the alleviation of chronic hepatitis C. Suggested mechanisms include the enhancement of a type $\mathrm{l}$ (Thl) responses by $\mathrm{T}$ cells as well as upregulation of MHC class I expression on cells. Both these mechanisms serve to aid viral clearance after infection and herpes virus has been proposed as an aetiological agent in Behçet's disease. Furthermore, interferon has recently been shown to inhibit $\gamma \delta+\mathrm{T}$ cells which have been implicated in the initial lesions of Behçet's disease. ${ }^{3}$ Paradoxically, there are reports of a Behçet's-like syndrome occurring in patients treated with the drug for chronic myeloid leukaemia, possibly by increasing neutrophil reactivity. Clearly, further work is required to elucidate the exact mechanisms involved.

\section{Behçet's disease has existed for thousands of years but we are still ignorant of its pathogenesis and treatment}

Other treatments for Behçet's disease are being tested. Currently, there is considerable interest in the potential role of anti-tumour necrosis factor (TNF) antibody therapy. TNF has been implicated in Behçet's disease, in that patients show higher serum levels of the cytokine than controls. TNF genetic polymorphisms that predispose to greater stimulated production of TNF from monocytes are associated with worse disease and drugs such as thalidomide and pentoxifyline, which are potent anti-TNF medications, are very effective in certain forms of the disease, particularly mucosal ulceration. Early results with the monoclonal antibody against TNF have shown benefit in ocular, ${ }^{4}$ orogenital, ${ }^{5}$ and gastrointestinal ${ }^{6}$ Behçet's disease, but long term efficacy is unknown.

Behçet's disease has existed for thousands of years but we are still ignorant of its pathogenesis and treatment. We do 
not know what causes it, why certain populations are more susceptible, why men tend to get worse disease, why it burns out in later life, and what is the best treatment. Much work remains to answer these questions and to devise specific therapy. However, new treatments are in development and hold promise for the future especially in those patients who do not respond to conventional therapy.

Br J Ophthalmol 2003;87:381-382

\section{Author's affiliations}

M R Stanford, Medical Eye Unit, St Thomas's Hospital, Lambeth Palace Road, London SE 1 7EH, UK; miles.stanford@kcl.ac.uk

\section{REFERENCES}

1 Muhaya $M$, Lightman S, Ikeda $E$, et al. Behçet's disease in Japan and Great Britain. Ocular Immunol Inflam 2000;8:141-8.

2 Alpsoy E, Durusoy C, Yilmaz E, et al. Interferon alfa-2a in the treatment of Behçet's disease: a randomised, placeobo controlled and double-blind study. Arch Dermatol 2002; 138:467-71
3 Hasan A Fortune F, Wilson $\mathrm{K}$, et al. Role of $\gamma \delta \mathrm{T}$ cells in the pathogenesis and diagnosis of Behçet's disease Lancet 1996;347:789-94.

4 Sfikakis PP. Theodossiadis PG Katsiari CG et al. Effect of infliximab on sight-threatening panuveitis in Behçet's disease. Lancet 2001;358:295-6.

5 Robertson LP, Hickling P. Treatment of recalcitrant orogenital ulceration of Behçet's syndrome with infliximab. Rheumatology 2001:40:473-4.

6 Travis SP, Czajkowski M, McGovern DP, et al. Treatment of intestinal Behçet's syndrome with chimeric tumour necrosis factor alpha antibody. Gut 2001;49:725-8.

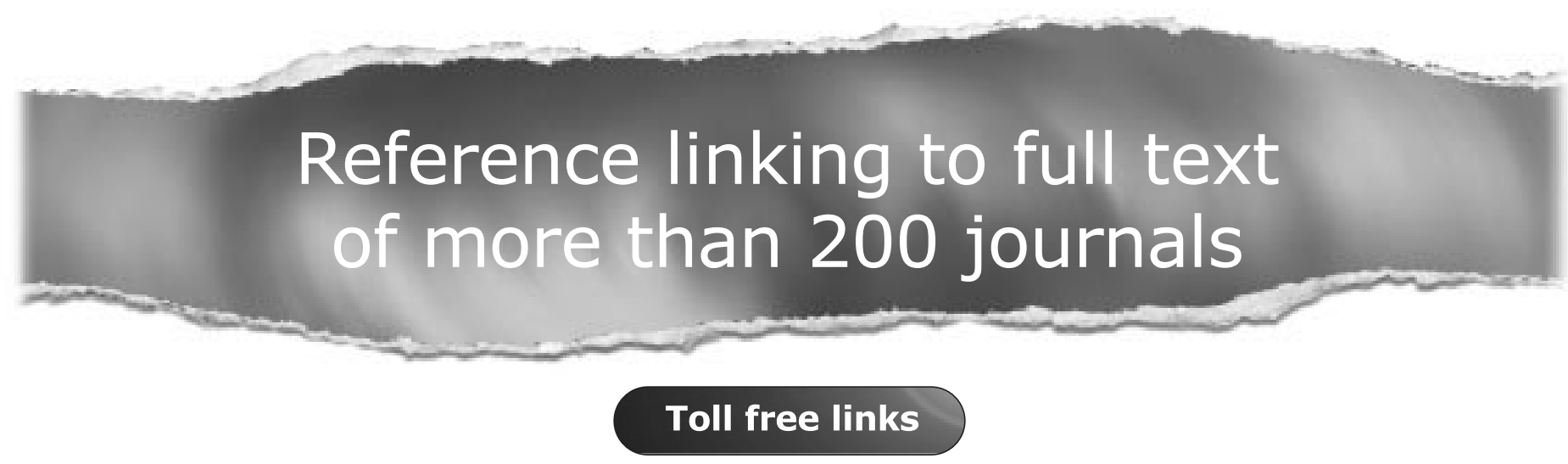

You can access the FULL TEXT of articles cited in the British Journal of Ophthalmology online if the citation is to one of the more than 200 journals hosted by HighWire (http://highwire.stanford.edu) without a subscription to that journal. There are also direct links from references to the Medline abstract for other titles.

www.bjophthalmol.com 